\title{
Eigenfrequencies and eigenmodes of a beam with periodically continuously varying spatial properties
}

\section{Sorokin, Vladislav S.; Thomsen, Jon Juel}

Published in:

Journal of Sound and Vibration

Link to article, DOI:

10.1016/j.jsv.2015.03.002

Publication date:

2015

Document Version

Peer reviewed version

Link back to DTU Orbit

Citation (APA):

Sorokin, V. S., \& Thomsen, J. J. (2015). Eigenfrequencies and eigenmodes of a beam with periodically continuously varying spatial properties. Journal of Sound and Vibration, 347, 14-26.

https://doi.org/10.1016/j.jsv.2015.03.002

\section{General rights}

Copyright and moral rights for the publications made accessible in the public portal are retained by the authors and/or other copyright owners and it is a condition of accessing publications that users recognise and abide by the legal requirements associated with these rights.

- Users may download and print one copy of any publication from the public portal for the purpose of private study or research.

- You may not further distribute the material or use it for any profit-making activity or commercial gain

- You may freely distribute the URL identifying the publication in the public portal

If you believe that this document breaches copyright please contact us providing details, and we will remove access to the work immediately and investigate your claim. 


\title{
Eigenfrequencies and eigenmodes of a beam with periodically continuously varying spatial properties
}

\author{
Vladislav S. Sorokin ${ }^{1,2}$, Jon Juel Thomsen ${ }^{1}$ \\ ${ }^{1}$ Department of Mechanical Engineering. Technical University of Denmark \\ Nils Koppels Allé, Building 404, 2800 Kgs. Lyngby, Denmark \\ ${ }^{2}$ Institute of Problems in Mechanical Engineering RAS \\ V.O., Bolshoj pr. 61, St.Petersburg, 199178, Russia \\ vladsor@mek.dtu.dk \\ phone: +4550121813 \\ fax: +4545251961
}

\begin{abstract}
A beam with periodically continuously varying spatial properties is analyzed. This structure is a generic model for various systems widely used in industry, e.g. risers, rotor blades, and similar. The aim is to reveal effects of periodic spatial modulation both on the beam eigenfrequencies and eigenmodes. Special attention is given to "mid-frequency" eigenmodes having period of the same order as the period of modulation, which cannot be captured by the conventional analytical methods. In particular, the paper addresses prediction of bandgaps and their influence on the distribution of eigenfrequencies. For analyzing the problem considered, the method of varying amplitudes is employed. A connection of this method with the classical Hill's infinite determinant method and the method of space-harmonics is noted.

A dispersion relation of the considered non-uniform periodic structure is obtained, and values of the modulation amplitudes at which frequency bandgaps arise are determined. It is shown that eigenfrequencies of the beam can lie within the bandgaps, and that such eigenfrequencies can be considerably affected by modulation. It is revealed that there is an abrupt shift in the effect of modulation on eigenfrequencies, and that modulations of the beam mass per unit length and of the beam stiffness affect them oppositely. It is shown that eigenmodes having a period close to the period of modulation comprise a long-wave component; this illustrates the capacity of non-uniform structures to sustain long-wave oscillations on comparatively high frequencies.
\end{abstract}

Keywords: eigenproperties; Bernoulli-Euler beam; periodic continuous spatial modulation; dispersion relation; bandgaps; method of varying amplitudes.

\section{Introduction}

Continua and structures composed of periodically repeated elements (cells) are used in many fields of science and technology. Examples of such continua are composite materials consisting of alternating volumes of substances with different properties, mechanical filters, and wave guides. Various frame structures, e.g. building frames and trusses of bridges, cranes and industrial constructions, railway tracks and compound pipes are also periodic systems. Design of these systems is challenged by the requirements to keep their response to time-dependent excitation 
within strictly prescribed limits. Depending on application, this response is controlled by frequency pass/stop bands, wave speeds, attenuation levels, eigenfrequencies, and eigenmodes.

The present paper is concerned with the analysis of a comparatively simple periodic structure, namely a beam with periodically continuously varying spatial properties. This structure, however, is a generic model for various more complicated systems, e.g. risers, rotor blades, and similar. Analysis of eigenfrequencies of non-uniform beams and rods is an old issue, see e.g. [1-3]. A profound review of papers in the field may be found in [4]. As follows from [4], most of the publications are devoted to the analysis of tapered beams and rods [5-8]. In particular, the exact solutions have been found for some specific cases of tapering [9-12]. On the other hand, several approximate analytical methods for determining the eigenfrequencies of non-uniform beams and rods were developed in $[4,13,14]$. In particular, the WKB method [15] was proposed for the analysis of spatially slowly varying beams and rods [16-18]. In contrast, the averaging procedure for processes in periodic structures described in [19] can be used to study spatially rapidly varying systems. However, these analytical approaches are applicable only in the presence of a small parameter in the governing equations. So, either the amplitude of modulation should be small [4], or the variation of structural parameters should be slow [16-18] or rapid [19], or a certain parameter (e.g. the thickness) of the corresponding homogeneous structure should be small [14]. In the present paper none of these restrictions are imposed, meaning that in particular the "mid-frequency" eigenmodes, with a period of the same order as the period of modulation, are considered. Analysis of such eigenmodes and eigenfrequencies is of particular importance for applications (e.g. $[18,20,21])$, though efficient tools for this are yet to be developed.

In contrast to the majority of the abovementioned papers, the focus of the present work is not only on eigenfrequencies, but also on eigenmodes of the considered non-uniform beam. Thus the aim is to reveal the effects of periodic modulation both on eigenfrequencies and eigenmodes. In particular, the paper addresses the prediction of bandgaps [22] and their influence on the distribution of eigenfrequencies. For analyzing the problem considered a novel approach named the method of varying amplitudes (MVA), proposed in [23], is employed. In [23] the dynamics of a string under the action of an external distributed time-periodic load was studied, and optimal parameters of the strings cross-sectional area were determined so as to suppress vibration in predefined regions of the string. By contrast to [23], the present work considers free oscillations of a Bernoulli-Euler beam, implying solving a fourth order differential equation, with the aim to reveal the effect of modulation on the dispersion relation and the eigenproperties. The MVA is inspired by the method of direct separation of motions (MDSM) [24,25], and may be considered a natural continuation of the classical methods of harmonic balance [26] and averaging [27-29]. It implies representing a solution in the form of a harmonic series with varying amplitudes, but in contrast to the averaging methods the amplitudes are not required to vary slowly. Thus the MVA does not assume the presence of a small parameter in the governing equations, or any restrictions on the sought solution. This, in particular, makes it convenient for analysis of the considered problem, which implies solving differential equations without a small parameter. It should be noted that the obtained solution obeys the Floquet theory [22]; and the method employed is connected with the classical Hill's infinite determinant method [22, 26, 30], and with the method of space-harmonics [31]. 


\section{Governing equations}

Consider oscillations of a Bernoulli-Euler beam with continuously varying spatial properties. The corresponding governing equation is:

$$
\rho A(\tilde{x}) \frac{\partial^{2} w}{\partial t^{2}}+\frac{\partial^{2}}{\partial \tilde{x}^{2}}\left(E I(\tilde{x}) \frac{\partial^{2} w}{\partial \tilde{x}^{2}}\right)=0,
$$

where $w(\tilde{x}, t)$ is the transverse displacement of the beam at time $t$ and axial coordinate $\tilde{x} \in[0 ; l], \rho$ is the density, $E$ is the Young's modulus of the beam material, $A$ the cross-sectional area, and $I$ the moment of inertia of the cross-section. Note that here only waves whose length is much larger than the height of the beam are considered, so that the classical Bernoulli-Euler theory holds and rotary inertia and shear deflections can be ignored. Dissipation is assumed to be negligibly small. The beam mass per unit length $\rho A(\tilde{x})$ and stiffness $E I(\tilde{x})$ are assumed to be periodically varying in the axial coordinate $\tilde{x}$ :

$$
\rho A(\tilde{x})=\rho A(\tilde{x}+\Theta), E I(\tilde{x})=E I(\tilde{x}+\Theta),
$$

where $\Theta$ is period of modulation. Note that $I(\tilde{x})=(r(\tilde{x}))^{2} A(\tilde{x})$, where $r(\tilde{x})$ is the radius of gyration of the cross-sectional area $A(\tilde{x})$. Expanding $\rho A(\tilde{x})$ and $E I(\tilde{x})$ in a trigonometric Fourier series gives:

$$
\begin{aligned}
& \rho A(\tilde{x})=\rho A_{0}\left[1+\sum_{m=1}^{\infty} \chi_{A, m} \sin \left(\frac{2 \pi}{\Theta} m \tilde{x}+\phi_{A, m}\right)\right], \\
& E I(\tilde{x})=E I_{0}\left[1+\sum_{m=1}^{\infty} \chi_{I, m} \sin \left(\frac{2 \pi}{\Theta} m \tilde{x}+\phi_{I, m}\right)\right]
\end{aligned}
$$

As an illustrative example a simply supported beam is studied, i.e. the boundary conditions are:

$$
\left.w\right|_{\tilde{x}=0}=\left.\frac{\partial^{2} w}{\partial \tilde{x}^{2}}\right|_{\tilde{x}=0}=\left.w\right|_{\tilde{x}=l}=\left.\frac{\partial^{2} w}{\partial \tilde{x}^{2}}\right|_{\tilde{x}=l}=0,
$$

where $l$ is the length of the beam. Other boundary conditions, in particular elastic boundaries, can be also considered by means of the approach employed in the paper; conditions (4) are chosen in order to illustrate this approach in the clearest and simplest manner.

The classical separation of variables technique is used to solve equation (1), i.e.:

$$
w(\tilde{x}, t)=\tilde{\Phi}(\tilde{x}) e^{i \omega t},
$$

where for the new variable $\tilde{\Phi}(\tilde{x})$ the following equation is obtained:

$$
E I_{0} \frac{d^{2}}{d \tilde{x}^{2}}\left(\left[1+\sum_{m=1}^{\infty} \chi_{I, m} \sin \left(m \frac{2 \pi}{\Theta} \tilde{x}+\phi_{I, m}\right)\right] \frac{d^{2} \tilde{\Phi}}{d \tilde{x}^{2}}\right)-\omega^{2} \rho A_{0}\left[1+\sum_{m=1}^{\infty} \chi_{A, m} \sin \left(m \frac{2 \pi}{\Theta} \tilde{x}+\phi_{A, m}\right)\right] \tilde{\Phi}=0,(6)
$$

where $\omega$ is the eigenfrequency of the considered non-uniform beam to be determined. Our aim is to reveal the principle effects of periodic modulation on the dispersion relation and eigenproperties of the beam. Consequently, to simplify the results, only the fundamental harmonic is accounted for in the series (3), so that (6) reduces to: 


$$
E I_{0} \frac{d^{2}}{d \tilde{x}^{2}}\left(\left(1+\chi_{I} \sin (k \tilde{x}+\phi)\right) \frac{d^{2} \tilde{\Phi}}{d \tilde{x}^{2}}\right)-\omega^{2} \rho A_{0}\left(1+\chi_{A} \sin (k \tilde{x}+\phi)\right) \tilde{\Phi}=0,
$$

where $0 \leq \chi_{A}<1,0 \leq \chi_{I}<1$, and in the simplest case of constant $\rho, E$, and $r$ we have $\chi_{A}=\chi_{I}$. Here, however, a more general case is considered, and the modulation amplitudes $\chi_{A}$ and $\chi_{I}$ are not required to be equal. Note that modulations of the beam mass per unit length and stiffness have the same phase shift $\phi$.

The MVA can be employed to solve (6) directly, so that the case of arbitrary modulation, relevant for real structures, can be also captured. Here, however, a more simple case is considered in order to illustrate the method and reveal principle effects arising in periodic BE beams.

Introducing a new variable $x=k \tilde{x}+\phi$ we rewrite equation (7) in dimensionless form:

$$
\left(\left(1+\chi_{I} \sin x\right) \Phi^{\prime \prime}\right)^{\prime \prime}-\delta\left(1+\chi_{A} \sin x\right) \Phi=0,
$$

where $\Phi(x)=\frac{\tilde{\Phi}(x)}{l}, \delta=\frac{\omega^{2} \rho A_{0}}{E I_{0} k^{4}}$, and primes denote derivatives with respect to $x$.

The boundary conditions for $\Phi(x)$ take the form:

$$
\Phi(\phi)=\Phi^{\prime \prime}(\phi)=\Phi(k l+\phi)=\Phi^{\prime \prime}(k l+\phi)=0 .
$$

To simplify the resulting expressions we further assume $\delta=O(1)$ (which comprises also the case $\delta<<1$ ); the case of large $\delta$ can be analyzed similarly. That means that only those waves whose period is of the same order as or much larger than period of modulation will be considered here. Note that this agrees well with the applicability range of the Bernoulli-Euler theory, which is not valid for high eigenfrequencies.

\section{Solution by the method of varying amplitudes}

To solve (8)-(9), which does not contain a small parameter since $\delta=O(1)$ and $0 \leq \chi_{A}<1$, $0 \leq \chi_{I}<1$, we employ a novel approach named the method of varying amplitudes (MVA) [23]. Following this method a solution of (8) is sought in the form of a harmonic series with varying amplitudes:

$$
\Phi(x)=b_{0}(x)+b_{11}(x) \cos x+b_{12}(x) \sin x+b_{21}(x) \cos 2 x+b_{22}(x) \sin 2 x+\ldots,
$$

where the amplitudes $b_{0}(x), b_{j 1}(x), b_{j 2}(x), j=1,2, \ldots m$, are not required to vary slowly in comparison with $\cos x, \cos 2 x$ etc. The shift from the original dependent variable $\Phi(x)$ to $2 m+1$ new variables $b_{0}(x), b_{j 1}(x), b_{j 2}(x)$ implies that $2 m+1$ equations for these variables are needed. This can be accomplished by introducing constraints in the form of $2 m$ additional equations. With the MVA the constraints are introduced in the following way: substitute (10) into (8), and require $2 m$ groups of terms to equal zero. The final $2 m+1$ 'th equation will include all the remaining terms of the original equation. The $2 m$ groups of terms are proposed to be the coefficients of the involved spatial harmonics, i.e. $\cos x, \sin x, \cos 2 x$ etc. Taking into account only the written terms in (10), one obtains five equations for the amplitudes $b_{0}(x), b_{11}(x), b_{12}(x), b_{21}(x), b_{22}(x)$ :

$$
b_{0}^{\prime \prime \prime \prime}-\delta b_{0}-\frac{\delta \chi_{A}}{2} b_{12}-\frac{\chi_{I}}{2} b_{12}^{\prime \prime}-\chi_{I} b_{11}^{\prime \prime \prime}+\frac{\chi_{I}}{2} b_{12}^{\prime \prime \prime}=0,
$$




$$
\begin{aligned}
& b_{11}^{\prime \prime \prime}-6 b_{11}^{\prime \prime}+(1-\delta) b_{11}+\left(2 \chi_{I}-\frac{\delta \chi_{A}}{2}\right) b_{22}-4 b_{12}^{\prime}+\chi_{I}\left(6 b_{21}^{\prime}-\frac{13}{2} b_{22}^{\prime \prime}-3 b_{21}^{\prime \prime \prime}+\frac{b_{22}^{\prime \prime \prime}}{2}\right)+4 b_{12}^{\prime \prime \prime}+2 \chi_{I} b_{0}^{\prime \prime \prime}=0,(12 \\
& b_{12}^{\prime \prime \prime}-6 b_{12}^{\prime \prime}+(1-\delta) b_{12}-\left(2 \chi_{I}-\frac{\delta \chi_{A}}{2}\right) b_{21}+4 b_{11}^{\prime}+\chi_{I}\left(6 b_{22}^{\prime}+\frac{13}{2} b_{21}^{\prime \prime}-3 b_{22}^{\prime \prime \prime}-\frac{b_{21}^{\prime \prime \prime}}{2}\right) \\
& -4 b_{11}^{\prime \prime \prime}-\delta \chi_{A} b_{0}-\chi_{I} b_{0}^{\prime \prime}+\chi_{I} b_{0}^{\prime \prime \prime \prime}=0 \\
& b_{21}^{\prime \prime \prime}+8 b_{22}^{\prime \prime \prime}-24 b_{21}^{\prime \prime}-32 b_{22}^{\prime}-(\delta-16) b_{21}+\left(\frac{\delta \chi_{A}}{2}-2 \chi_{I}\right) b_{12}+\chi_{I}\left(-6 b_{11}^{\prime}+\frac{13}{2} b_{12}^{\prime \prime}+3 b_{11}^{\prime \prime \prime}-\frac{1}{2} b_{12}^{\prime \prime \prime}\right)=0, \\
& {\left[b_{22}^{\prime \prime \prime}-8 b_{21}^{\prime \prime \prime}-24 b_{22}^{\prime \prime}+32 b_{21}^{\prime}-(\delta-16) b_{22}-\left(\frac{\delta \chi_{A}}{2}-2 \chi_{I}\right) b_{11}+\chi_{I}\left(-6 b_{12}^{\prime}-\frac{13}{2} b_{11}^{\prime \prime}+3 b_{12}^{\prime \prime \prime}+\frac{1}{2} b_{11}^{\prime \prime \prime}\right)\right] \sin 2 x} \\
& =-\left[\left(18 \chi_{I}-\frac{\delta \chi_{A}}{2}\right) b_{21}+\chi_{I}\left(\frac{1}{2} b_{21}^{\prime \prime \prime}+5 b_{22}^{\prime \prime \prime}-30 b_{22}^{\prime}-\frac{37}{2} b_{21}^{\prime \prime}\right)\right] \sin 3 x \\
& +\left[\left(18 \chi_{I}-\frac{\delta \chi_{A}}{2}\right) b_{22}+\chi_{I}\left(\frac{1}{2} b_{22}^{\prime \prime \prime}-5 b_{21}^{\prime \prime \prime}+30 b_{21}^{\prime}-\frac{37}{2} b_{22}^{\prime \prime}\right)\right] \cos 3 x .
\end{aligned}
$$

Thus we have restated the original equation (8) in the form of five equations (11)-(15), with $b_{0}, b_{11}, b_{12}, b_{21}, b_{22}$ as the dependent variables instead of $\Phi$; no approximations are involved so far. Approximations are introduced by neglecting the right-hand side of (15), so that:

$$
b_{22}^{\prime \prime \prime}-8 b_{21}^{\prime \prime \prime}-24 b_{22}^{\prime \prime}+32 b_{21}^{\prime}-(\delta-16) b_{22}-\left(\frac{\delta \chi_{A}}{2}-2 \chi_{I}\right) b_{11}+\chi_{I}\left(-6 b_{12}^{\prime}-\frac{13}{2} b_{11}^{\prime \prime}+3 b_{12}^{\prime \prime \prime}+\frac{1}{2} b_{11}^{\prime \prime \prime}\right)=0,(16
$$

the simplification being valid when

$$
\left|18 \chi_{I}-\frac{\delta \chi_{A}}{2}\right|<<|\delta-16|,
$$

i.e. when the right-hand side of (15) is small in comparison with the leading terms of (14)-(15). Since $\delta=O(1)$, and $\chi_{A} \in[0 ; 1)$ an additional restriction $\chi_{I}<<1$ should be imposed (in fact it is sufficient to require $\chi_{I} \leq 0.5$, cf. Section 6 ). So, only comparatively small modulations of the beam stiffness can be considered by the method, while modulations of the beam mass per unit length can be large.

Note that the procedure of the method will not change if solving (6) instead of (7), but the number of harmonics taken into account in series (10) can increase, since the right-hand side of the last $2 m+1$ 'th equation should be small in comparison with the leading terms.

The general solution of equations (11)-(14), (16) may be written in the form $\mathbf{b}=\left\{\begin{array}{lllll}b_{0} & b_{11} & b_{12} & b_{21} & b_{22}\end{array}\right\}^{\mathrm{T}}:$

$$
\mathbf{b}=C_{1} \mathbf{b}_{1} \exp \lambda_{1} x+C_{2} \mathbf{b}_{2} \exp \lambda_{2} x+\ldots+C_{20} \mathbf{b}_{20} \exp \lambda_{20} x,
$$

where $\lambda_{j}(j=1, \ldots, 20)$ are roots of the characteristic equation corresponding to (11)-(14), (16), $\mathbf{b}_{j}$ the associated vectors which depend on the system parameters $\delta, \chi_{I}, \chi_{A}$, and $C_{1}, \ldots, C_{20}$ are arbitrary constants; note that boundary conditions are not yet employed. Consequently, taking into account (5), (10) and (18) we can write the general solution of the initial equation (1) in the form: $w(\tilde{x}, t)=\sum_{j=1}^{20} \tilde{C}_{j}\left(b_{0 j}+b_{11 j} \cos (k \tilde{x}+\phi)+b_{12 j} \sin (k \tilde{x}+\phi)+b_{21 j} \cos 2(k \tilde{x}+\phi)+b_{22 j} \sin 2(k \tilde{x}+\phi)\right) \exp \left(\lambda_{j} k \tilde{x}+i \omega t\right)$, 
where $b_{0 j}, b_{11 j}, b_{12 j}, b_{21 j}, b_{22 j}$ are corresponding elements of the vector $\mathbf{b}_{j}$ associated with root $\lambda_{j}$. Introducing a new variable $\tilde{\lambda}_{j}=i \lambda_{j} k$, a particular solution of (1) may be rewritten as:

$$
\begin{aligned}
& w_{j}(\tilde{x}, t)=F_{j}(\tilde{x}) \exp \left(i\left(\omega t-\tilde{\lambda}_{j} \tilde{x}\right)\right) \\
& F_{j}(\tilde{x})=b_{0 j}+b_{11 j} \cos (k \tilde{x}+\phi)+b_{12 j} \sin (k \tilde{x}+\phi)+b_{21 j} \cos 2(k \tilde{x}+\phi)+b_{22 j} \sin 2(k \tilde{x}+\phi)
\end{aligned},
$$

As appears the solution (20) obeys the Floquet theory [22], since the function $F_{j}(\tilde{x})$ has the same period as the modulation. Note also that in the general case, i.e. with infinite number of harmonics taken into account in the series (10), the solution form (20) would become similar to the one implied in the method of space-harmonics [31], which is an efficient method for determining dispersion relations of discontinuous periodic structures.

Also a connection of the MVA with the classical Hill's infinite determinant method [22, 26, 30], developed for analyzing Mathieu-Hill type equations, should be mentioned: both methods imply calculation of matrix determinants for obtaining expressions for the roots $\lambda_{j}$, and thus for the wave numbers $\tilde{\lambda}_{j}$. Note however, that the MVA has a much broader applicability range, e.g. it can be applied also for solving problems involving combined direct and parametric excitation [23], and for nonlinear problems [32]. Another important feature of the MVA is that it provides an explicit condition, similar to (17) for the present problem, under which the obtained solution is accurate.

As appears in the considered case the MVA requires the presence of a small parameter $\chi_{I}$ in the governing equation, similarly to the multiple scales method (MSM). This is due to the special case considered, where the coefficient of the highest derivative is modulated. For studying other cases of parametric excitation, e.g. the one considered in [23], the MVA can be employed without the presence of a small parameter. Note also that even when $\chi_{I}<<1$ the MSM and other asymptotic methods are not applicable for solving the considered equation (8), since $\chi_{A}$ is not small. Thus the applicability range of the MVA is broader than for these methods.

\section{Dispersion relation}

The dependency of the roots $\lambda_{j}$ on the parameter $\delta$ represents the dispersion relation of the considered periodic structure, since $\delta$ is proportional to squared frequency $\omega$, and the wave number $\tilde{\lambda}_{j}$ is equal to $\tilde{\lambda}_{j}=i \lambda_{j} k$. In the case without modulation $\left(\chi_{I}=0, \chi_{A}=0\right)$, i.e. with a uniform beam, the roots of the characteristic equation corresponding to (11)-(14), (16) are determined by $\lambda_{1-10}=i m \pm \sqrt[4]{\delta}, \lambda_{11-20}=i m \pm i \sqrt[4]{\delta}, m=-2,-1,0,1,2$, and the corresponding twenty particular solutions (20) of (1) can be reduced to just four generic solutions. To illustrate this, consider two roots $\lambda_{1}=-2 i+\sqrt[4]{\delta}$ and $\lambda_{2}=\sqrt[4]{\delta}$; the vector associated with the first root is $\mathbf{b}_{1}=\left\{\begin{array}{llll}0 & 0 & 0 & 1\end{array} i\right\}^{\mathbf{T}}$, and for the second root $\mathbf{b}_{2}=\left\{\begin{array}{lllll}1 & 0 & 0 & 0 & 0\end{array}\right\}^{\mathbf{T}}$. Then the particular solution (20) corresponding to the first root takes the form:

$$
w_{1}(\tilde{x}, t)=\exp (2 i k \tilde{x}) \exp \left(i\left(\omega t-i \lambda_{1} k \tilde{x}\right)\right)=\exp (i \omega t+\sqrt[4]{\delta} k \tilde{x})
$$

while for the second root:

$$
w_{2}(\tilde{x}, t)=\exp (i \omega t+\sqrt[4]{\delta} k \tilde{x})
$$


As appears they are identical. Thus it is adequate to consider only four generic roots of the characteristic equation corresponding to (11)-(14), (16), e.g. $\lambda_{1,2}= \pm \sqrt[4]{\delta}, \lambda_{3,4}= \pm i \sqrt[4]{\delta}$. By generic roots we understand those roots giving particular solutions which cannot be reduced to (or replaced by) each other. Taking into account that $\delta=\frac{\omega^{2} \rho A_{0}}{E I_{0} k^{4}}$ and $\tilde{\lambda}_{j}=i \lambda_{j} k$, we obtain the well-known dependencies of the wave number $\tilde{\lambda}$ on frequency $\omega$ for a uniform beam: $\tilde{\lambda}_{1,2}= \pm i \sqrt[4]{\rho A_{0} /\left(E I_{0}\right)} \sqrt{\omega}$ , $\tilde{\lambda}_{3,4}= \pm \sqrt[4]{\rho A_{0} /\left(E I_{0}\right)} \sqrt{\omega}$. Note that real generic roots $\lambda_{1,2}= \pm \sqrt[4]{\delta}$ of the characteristic equation correspond to attenuating waves, and imaginary roots $\lambda_{3,4}= \pm i \sqrt[4]{\delta}$ to propagating waves.

In the presence of spatial cross-section modulation, expressions for the roots of the characteristic equation corresponding to system (11)-(14), (16) become much more involved, and thus are not given here. The dependency of $\sqrt{\delta} \sim \omega$ on the real-valued generic roots $\lambda_{1 n}$ and $\lambda_{2 n}$ of the characteristic equation, corresponding to attenuating waves, is shown in Figure 1 for various values of the modulation amplitudes $\chi_{I}, \chi_{A}$. Note that for $\chi_{I}=0, \chi_{A}=0$ these roots reduce to $\lambda_{1,2}= \pm \sqrt[4]{\delta}$. As appears the branches are symmetric with respect to the vertical axis (i.e. $\lambda_{1 n}=-\lambda_{2 n}$ ), and only slightly perturbed due to modulation.

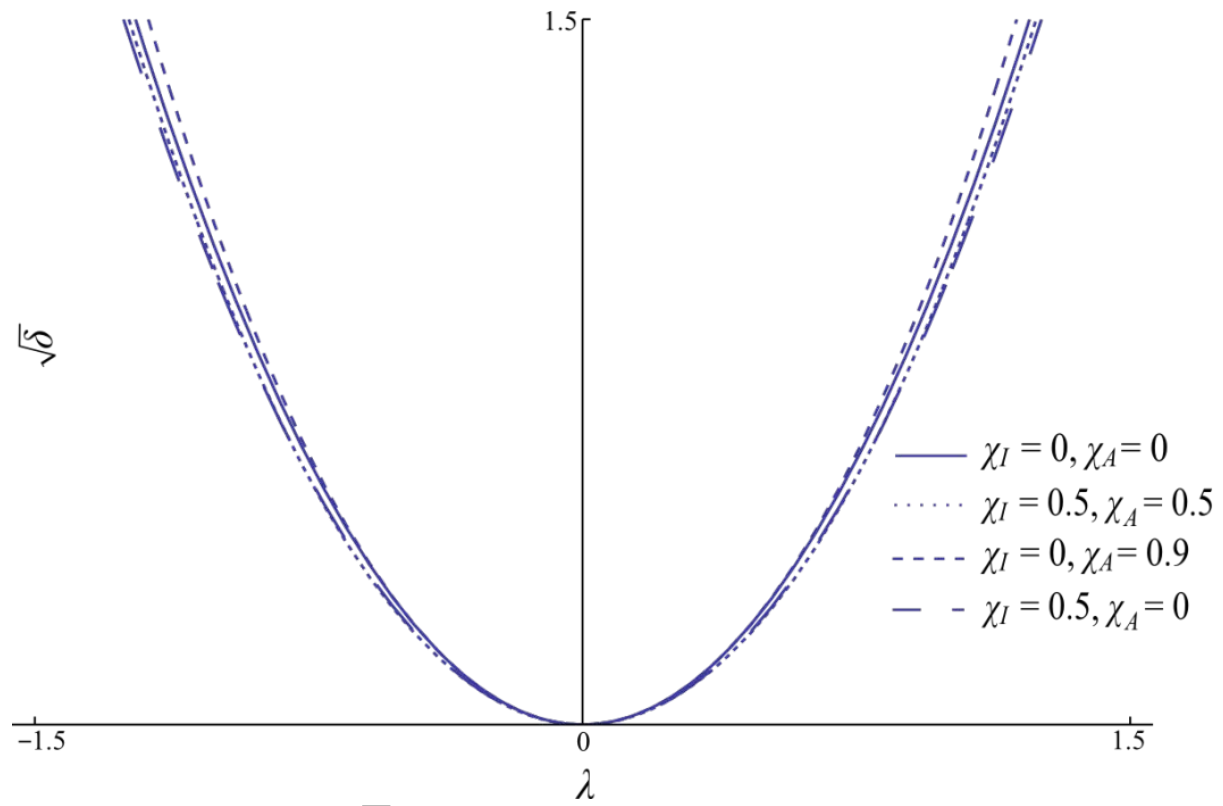

Figure 1 . The dependency of $\sqrt{\delta} \sim \omega$ on the real-valued roots $\lambda_{1 n}$ and $\lambda_{2 n}$ of the characteristic equation, corresponding to attenuating waves, for various values of the modulation amplitudes $\chi_{I}$, $\chi_{A}$.

More interesting effects are present for propagating waves, which correspond to imaginary roots $\lambda$ of the characteristic equation. As an illustration the dependencies of $\sqrt{\delta} \sim \omega$ on $i \lambda$ are shown in Figure 2 for various values of the modulation amplitudes $\chi_{I}, \chi_{A}$. The imaginary generic roots $\lambda_{3 n}$ and $\lambda_{4 n}$ of the characteristic equation are bounded by dotted lines, which in fact represent the first Brillouin zone [22]. According to [22], the dispersion relations of periodic structures can be 
presented only for this zone, since the frequency $\omega$ is a periodic function of the wave number $\tilde{\lambda}$. As appears this statement holds true for the dispersion relations shown in Figure 2, since $\sqrt{\delta}$, and thus the frequency $\omega$, is a 1-periodic function of imaginary roots $\lambda$, i.e. a $k$-periodic function of wave numbers $\tilde{\lambda}$. This also justifies the adequacy of considering only four generic roots of the characteristic equation corresponding to system (11)-(14), (16), implied in the MVA.
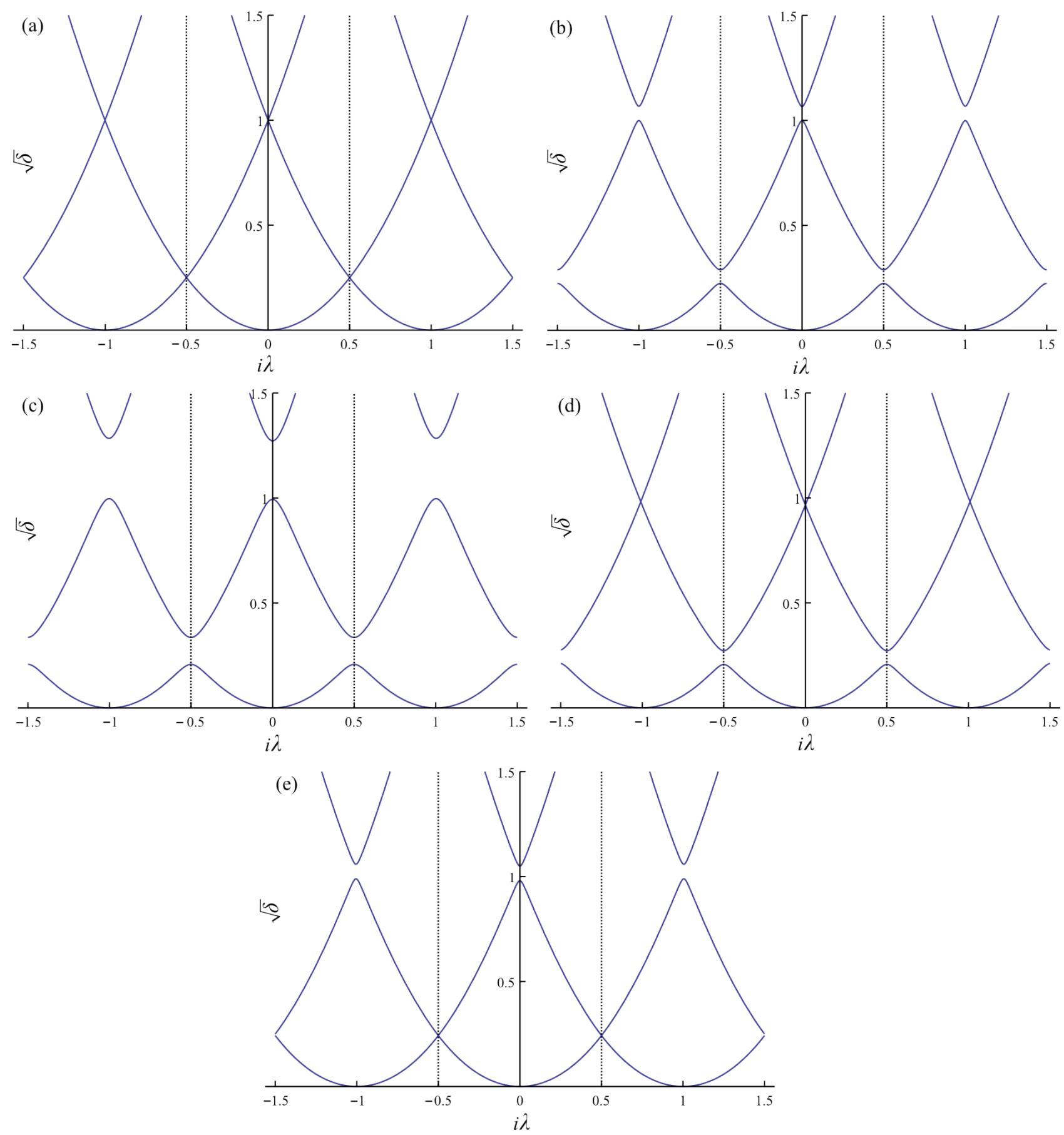

Figure 2. The dependencies of $\sqrt{\delta} \sim \omega$ on $i \lambda$ corresponding to propagating waves for (a) $\chi_{I}=0$, $\chi_{\mathrm{A}}=0$; (b) $\chi_{I}=0, \chi_{\mathrm{A}}=0.5$; (c) $\chi_{I}=0, \chi_{\mathrm{A}}=0.9$; (d) $\chi_{I}=0.5, \chi_{\mathrm{A}}=0$; (e) $\chi_{I}=0.5, \chi_{\mathrm{A}}=0.5$. 
As appears from Figure 2, at certain values of the modulation amplitudes frequency bandgaps arise, i.e. frequency ranges in which there can be no propagating waves. It is interesting to note that pure modulation $\chi_{A}$ of the beam mass per unit length leads to emergence of two distinct bandgaps in the considered frequency range (at $i \lambda=0.5$ and $i \lambda=0$ ), while pure modulation $\chi_{I}$ of the beam stiffness gives one bandgap (at $i \lambda=0.5$ ), and modulations with equal amplitudes $\chi_{\mathrm{A}}=\chi_{I}$ also one bandgap (at $i \lambda=0$ ). It turns out that the bandgaps affect distribution of the beam eigenfrequencies, as will be discussed next.

\section{Effect of modulation on eigenfrequencies and eigenmodes}

The general solution of (8) is obtained in the form:

$$
\Phi(x)=\sum_{j=1}^{4} C_{j}\left(b_{0 j}+b_{11 j} \cos x+b_{12 j} \sin x+b_{21 j} \cos 2 x+b_{22 j} \sin 2 x\right) \exp \left(\lambda_{j} x\right),
$$

where $\lambda_{j}$ is a generic root of the characteristic equation corresponding to the system (11)-(14), (16), and $\mathbf{b}_{j}=\left\{b_{0 j} b_{11 j} b_{12 j} b_{21 j} b_{22 j}\right\}^{\mathbf{T}}$ a constant vector corresponding to this root. Consequently, the boundary conditions (9) are rewritten as

$$
\begin{aligned}
& \Phi(\phi)=\sum_{j=1}^{4} C_{j}\left(b_{0 j}+b_{11 j} \cos \phi+b_{12 j} \sin \phi+b_{21 j} \cos 2 \phi+b_{22 j} \sin 2 \phi\right) \exp \left(\lambda_{j} \phi\right)=0, \\
& \Phi(k l+\phi)=\sum_{j=1}^{4} C_{j}\left(b_{0 j}+b_{11 j} \cos (k l+\phi)+b_{12 j} \sin (k l+\phi)+b_{21 j} \cos 2(k l+\phi)+b_{22 j} \sin 2(k l+\phi)\right) \exp \left(\lambda_{j}(k l+\phi)\right)=0, \\
& \Phi^{\prime \prime}(\phi)=\sum_{j=1}^{4} C_{j}\left[\lambda_{j}^{2} b_{0 j}+\left(\left(\lambda_{j}^{2}-1\right) b_{11 j}+2 \lambda_{j} b_{12 j}\right) \cos \phi+\left(\left(\lambda_{j}^{2}-1\right) b_{12 j}-2 \lambda_{j} b_{11 j}\right) \sin \phi+\left(\left(\lambda_{j}^{2}-4\right) b_{21 j}+4 \lambda_{j} b_{22 j}\right) \cos 2 \phi\right. \\
& \left.+\left(\left(\lambda_{j}^{2}-4\right) b_{22 j}-4 \lambda_{j} b_{21 j}\right) \sin 2 \phi\right] \exp \left(\lambda_{j} \phi\right)=0, \\
& \Phi^{\prime \prime}(k l+\phi)=\sum_{j=1}^{4} C_{j}\left[\lambda_{j}^{2} b_{0 j}+\left(\left(\lambda_{j}^{2}-1\right) b_{11 j}+2 \lambda_{j} b_{12 j}\right) \cos (k l+\phi)+\left(\left(\lambda_{j}^{2}-1\right) b_{12 j}-2 \lambda_{j} b_{11 j}\right) \sin (k l+\phi)\right. \\
& \left.+\left(\left(\lambda_{j}^{2}-4\right) b_{21 j}+4 \lambda_{j} b_{22 j}\right) \cos 2(k l+\phi)+\left(\left(\lambda_{j}^{2}-4\right) b_{22 j}-4 \lambda_{j} b_{21 j}\right) \sin 2(k l+\phi)\right] \exp \left(\lambda_{j}(k l+\phi)\right)=0 .
\end{aligned}
$$

The eigenfrequencies of the considered non-uniform beam are then determined using relations (24). First the constants $C_{4}, C_{3}, C_{2}$ are expressed via $C_{1}$ using three boundary conditions (the corresponding expressions for these constants being too lengthy to be given here). Employing then these expressions, and remembering that roots $\lambda_{j}$ are functions of $\delta$, which is proportional to squared eigenfrequency $\omega^{2}$, we compose a transcendental equation for $\delta$ :

$$
D\left(\delta, \chi_{I}, \chi_{A}, k l, \phi\right)=0
$$

Subsequently, having determined the eigenfrequencies, we obtain the eigenmodes of the considered non-uniform beam in the form (23). To further analyze the effect of modulation on the beam eigenproperties two different cases will be considered separately: 1) modulation with zero mean, i.e. $k=2 p \frac{\pi}{l}, p=1,2,3 \ldots$, and 2) modulation with non-zero mean, i.e. $k \neq 2 p \frac{\pi}{l}$.

\subsection{Case: Zero mean cross-section modulation}

In this case the effect of modulation on a certain eigenfrequency strongly depends on the index of this eigenfrequency. For example lower eigenfrequencies are changed only slightly due to 
modulation of the beam mass per unit length (with amplitude $\chi_{\mathrm{A}}$ ), whereas higher eigenfrequencies increase considerably. As an illustration the relative changes of the first five eigenfrequencies are presented in Figure 3 for:

$$
k=2 p \frac{\pi}{l} \mathrm{~cm}^{-1}, p=2, l=1 \mathrm{~cm}, \frac{\rho A_{0}}{E I_{0}}=1 \mathrm{~s}^{2} / \mathrm{cm}^{4}, \phi=0,
$$

and various values of the modulation amplitudes $\chi_{A}$, $\chi_{I}$. Here $\omega_{n 0}$ and $\omega_{n}$ is the nth eigenfrequency of, respectively, the uniform and the non-uniform beams. Note that $I(\tilde{x})=(r(\tilde{x}))^{2} A(\tilde{x})$, so the considered values of $\chi_{A}$ and $\chi_{I}$ correspond to different variations of $E, \rho, r$ and $A$, e.g. $\chi_{A}=0$ and $\chi_{I} \neq 0$ correspond to constant $\rho, E$, and $A$, and non-constant $r$. As appears from Figure 3(a), the first two eigenfrequencies are changed only slightly due to modulation of the beam mass per unit length $\chi_{A}$, whereas higher eigenfrequencies increase considerably.

The result for lower eigenfrequencies is in good agreement with [19] for a beam with rapidly varying cross-section: modulation of the beam mass per unit length does not affect eigenfrequencies of standing waves whose spatial period is much higher than the period of modulation. However, the revealed effect for higher eigenfrequencies is noted, apparently, for the first time, since it is for waves whose period is of the same order as the period of modulation, and the existing analytical methods [14-19] are not applicable for analyzing such eigenproperties. Note however, that for parameters (26) only the first eigenmode has a period which is much higher than the period of modulation (since period of the second eigenmode is $l$ ).

Another interesting effect noted for the first time, apparently, is that modulation $\chi_{I}$ of the beam stiffness considerably decreases both lower and higher eigenfrequencies, cf. Figure 3(b). Thus, if both modulations $\chi_{I}$ and $\chi_{A}$ are imposed, the higher beam eigenfrequencies, which correspond to eigenmodes with a period of the same order as the period of modulation, can be either reduced or increased; this is illustrated in Figure 3(c) by the third, the fourth, and the fifth eigenfrequencies.

The revealed effects clearly indicate the capability of cross-section modulation to change spacing between eigenfrequencies. For example, imposing a strong modulation $\chi_{A}$ of the beam mass per unit length for parameters (26), the spacing between the second and the third eigenfrequencies can be considerably increased. These results are in good agreement with those obtained numerically, e.g. in [21], where the task of maximizing the spacing between two adjacent eigenfrequencies was considered.

Considering the influence of bandgaps on the beam eigenfrequencies two notable effects appear: 1) Eigenfrequencies can lie within the bandgaps; 2) such eigenfrequencies can be changed significantly by cross-section modulation. For example in Figure 3(a) the second and the fourth eigenfrequency are within the bandgaps; as is seen, the fourth eigenfrequency is increased rather dramatically. In Figure 3(b) the second eigenfrequency is within the bandgap, and is decreased significantly. 

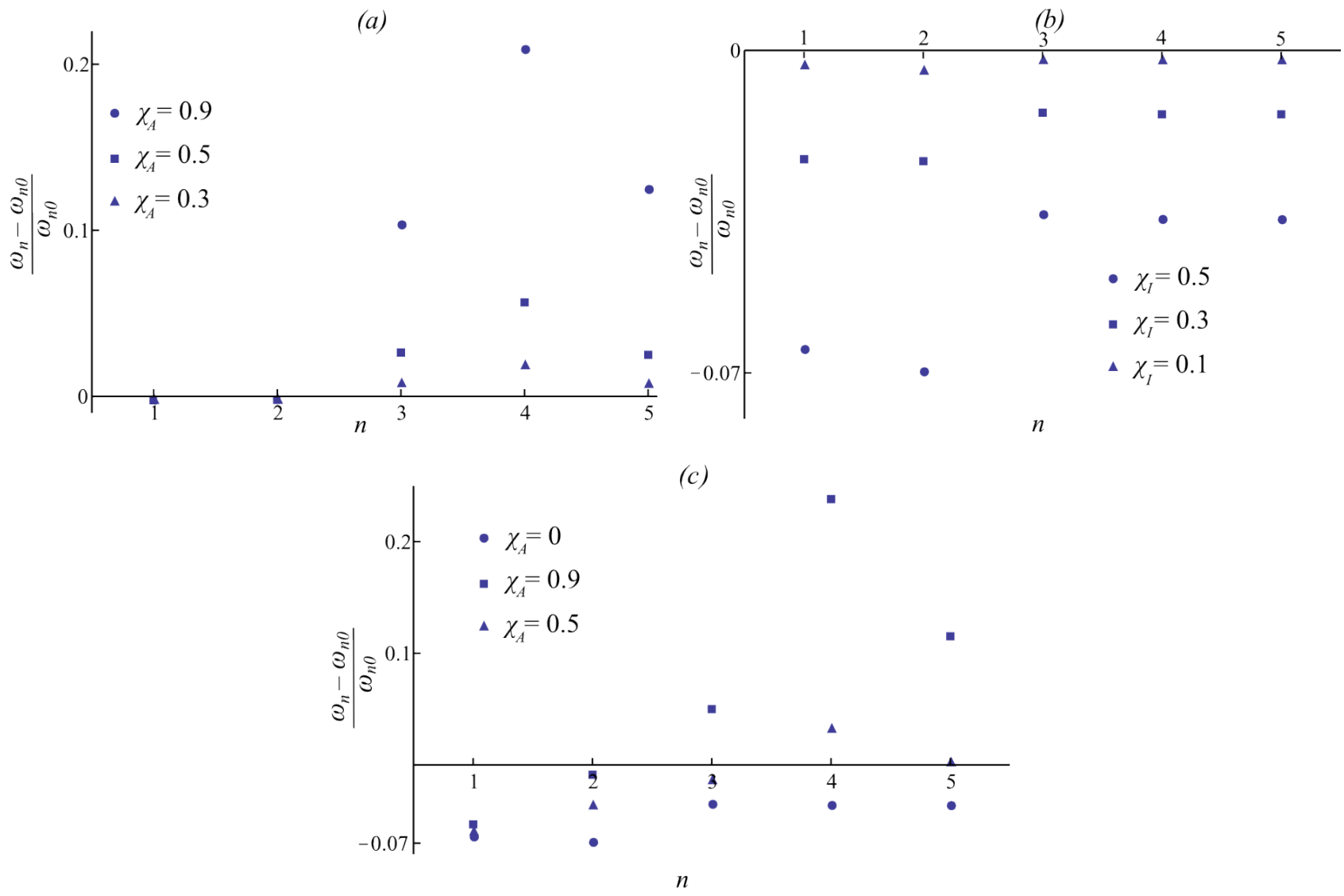

Figure 3. Relative change of the first five eigenfrequencies for parameters (26) and (a) $\chi_{I}=0$,

$$
\text { (b) } \chi_{\mathrm{A}}=0 \text {, and (c) } \chi_{I}=0.5 \text {. }
$$

Now consider the effect of modulation on the eigenmodes of the beam. As an illustration, in Figure 4 the first five eigenmodes are presented for parameters (26) and various values of $\chi_{I}$ and $\chi_{A}$ (solid lines); the eigenmodes of the corresponding uniform beam are also shown for comparison (dashed lines). As is seen, modulation considerably affects the eigenmodes, e.g., though not influencing the second eigenfrequency, modulation $\chi_{A}$ of the beam mass per unit length significantly changes the corresponding eigenmode (Figure 4 (b)).

Another interesting effect noted for the first time, apparently, is presence of a long-wave component in eigenmodes whose period is close to the period of modulation, see e.g. figures 4 (c), (d) and (e); the component is shown by dotted lines. Though this component is not equal to zero on boundaries, the eigenmodes (solid lines) obey the boundary conditions. This effect can be explained by considering the obtained expression (23) for the eigenmodes and the dispersion relations presented in Figure 2. As appears imaginary roots $i \lambda$ (i.e. wave numbers) corresponding to comparatively high frequencies $\sqrt{\delta} \approx 1$ are relatively small. Thus the eigenmodes (23) corresponding to eigenfrequencies from this range should involve small values of $\lambda_{j}$, which in turn means that a long-wave component $b_{0 j} \exp \left(\lambda_{j} x\right)$ (here $\lambda_{j}$ is imaginary) should be present in these 
eigenmodes. On the other hand they also include components $b_{11 j} \cos x \exp \left(\lambda_{j} x\right)$ and $b_{12 j} \sin x \exp \left(\lambda_{j} x\right)$, indicating a period close to the period of modulation.

This effect seems to be rather important in illustrating the potential of non-uniform structures to sustain long-wave oscillations at comparatively high frequencies. A similar effect was revealed in [33] where free oscillations of a non-uniform string were considered.
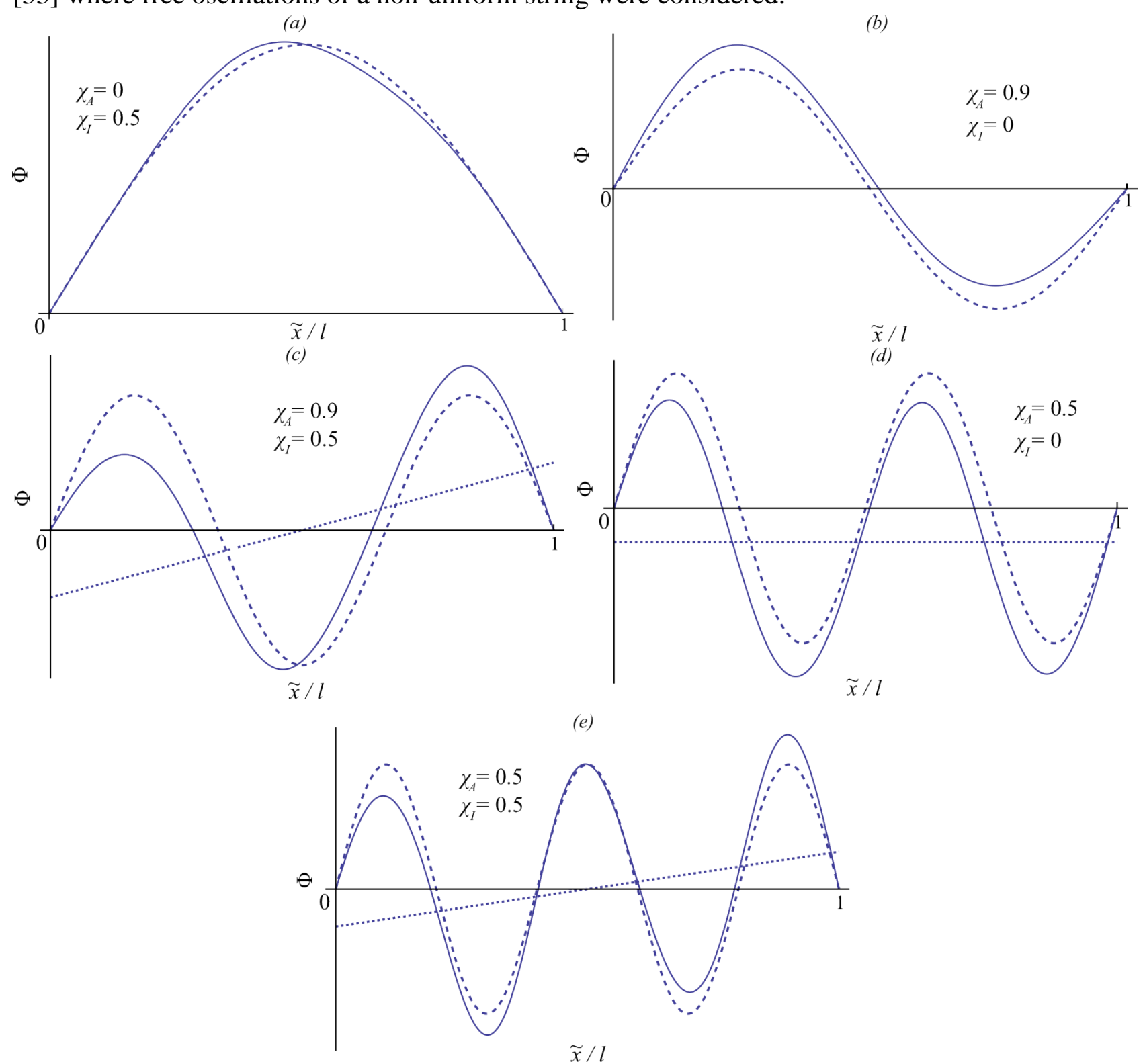

Figure 4. The first five eigenmodes of a non-uniform (solid lines) and a corresponding uniform (dashed lines) beam for parameters (26) and various values of the modulation amplitudes $\chi_{A}, \chi_{I}$.

Dotted lines represent the long-wave component of the third, fourth, and fifth eigenmodes.

\subsection{Case: Non-zero mean cross-section modulation}

Now consider modulation which has non-zero mean. In this case, as in Section 5.1, the effect of modulation on a certain eigenfrequency strongly depends on the index of the eigenfrequency. For 
illustration, in Figure 5 the relative changes of the first nine eigenfrequencies of the beam are presented for

$$
k=(2 p-1) \frac{\pi}{l} \mathrm{~cm}^{-1}, p=5, l=1 \mathrm{~cm}, \frac{\rho A_{0}}{E I_{0}}=1 \mathrm{~s}^{2} / \mathrm{cm}^{4}, \phi=0,
$$

and various values of the modulation amplitudes $\chi_{A}, \chi_{I}$. As appears from Figure 5(a), modulation $\chi_{A}$ of the beam mass per unit length increases the first four eigenfrequencies, the change of the fourth being most pronounced, but decreases the next three eigenfrequencies, especially the fifth. Note, however, that in this case only the first three eigenmodes have a period which is much higher than the period of modulation.

Thus there is an abrupt shift in the effect of this modulation on higher eigenfrequenies. A similar shift is present also in the variation of the eigenfrequencies due to modulation $\chi_{I}$ of the beam stiffness. For example for parameters (27) this modulation decreases the first four eigenfrequencies, but increases the fifth, cf. Figure 5(b).

For other values of $p$, the index $n$ of the spacing between eigenfrequencies $\omega_{n}$ and $\omega_{n+1}$, for which the effect of modulation sharply changes, turns out to be $n=p-1$. So this occurs for eigenfrequencies $\omega_{n}$ and $\omega_{n+1}$ corresponding to wavelengths closest to twice the modulation period, i.e. $2 \times 2 \pi / k$. For example, for $p=3$ and other parameters defined by (27), modulation $\chi_{A}$ of the beam mass per unit length increases the first two eigenfrequencies, the change of the second being most pronounced, but decreases the third eigenfrequency.

Thus the spacing between eigenfrequencies $\omega_{n}$ and $\omega_{n+1}$ can be changed considerably by means of cross-section modulation. However, if equal modulations are imposed $\left(\chi_{A}=\chi_{I}\right)$, no abrupt shift in the effect of modulation on eigenfrequencies can be noticed (cf. Figure 5) (recall that $\chi_{\mathrm{A}}=\chi_{I}$ corresponds to the important case of constant $\rho, E$, and $r$ ).

As for the case considered in Section 5.1 we find that eigenfrequencies within the bandgaps are considerably affected by modulation. For example, in Figure 5(a) the fifth and the ninth eigenfrequencies are within the gaps, and changing significantly, and in Figure 5(c) the ninth eigenfrequency lies in bandgap. However, in the case presented in Figure 5(b) none of the eigenfrequencies are in a bandgap, though they are considerably changed by modulation.

These results, and those presented in the previous section, indicate that the eigenfrequency corresponding to an eigenmode with a period equal to the modulation period, i.e. the fourth one for parameters (26) and the ninth one for parameters (27), is always within the bandgap for $\chi_{A} \neq 0$, and is increased considerably by modulation. 

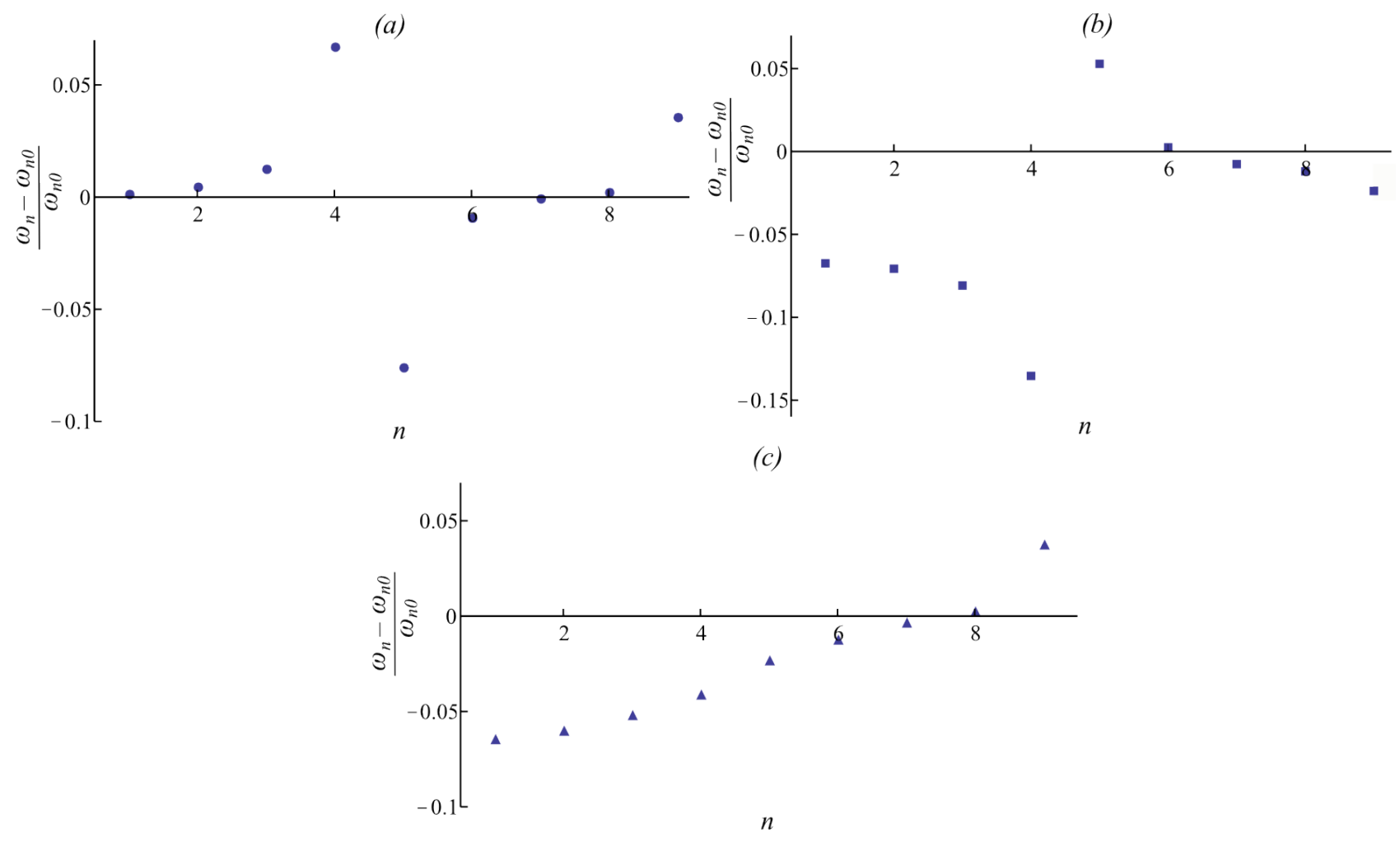

Figure 5. Relative change of the first nine eigenfrequencies for parameters (27) and (a) $\chi_{A}=0.5$,

$$
\chi_{I}=0 \text {; (b) } \chi_{\mathrm{A}}=0, \chi_{I}=0.5 \text {; (c) } \chi_{\mathrm{A}}=0.5, \chi_{I}=0.5 \text {. }
$$

Examining the effect of cross-section modulation on the eigenmodes of the beam, we again identify the emergence of a long-wave component in modes having a period close to the modulation period; this can be explained in a similar way as in the previous section. For illustration, Figure 6 presents the sixth, seventh, eighth, and ninth eigenmodes of the beam for parameters (27) and various values of $\chi_{A}, \chi_{I}$; the long-wave component is shown by dashed lines. It is interesting to note that pure modulation $\chi_{I}$ of the beam stiffness does not produce a long-wave component, cf. Figure 6(e); note also that this modulation does not imply gaps in the dispersion relations at $i \lambda=0$, cf. Figure 2(d).

It turns out that an eigenmode with a period equal to the modulation period has a long-wave component which is almost constant, cf. Figure 6(d). This follows from the fact that this eigenmode, determined by expression (23), involves a very small value of $\lambda_{j}$.

The effects revealed and explained in this section, and also in Section 4, are general in the sense that they can be present not only for the considered case of harmonic spatial modulation, but also for an arbitrary periodic modulation, which is the case particularly relevant for applications. 
(a)

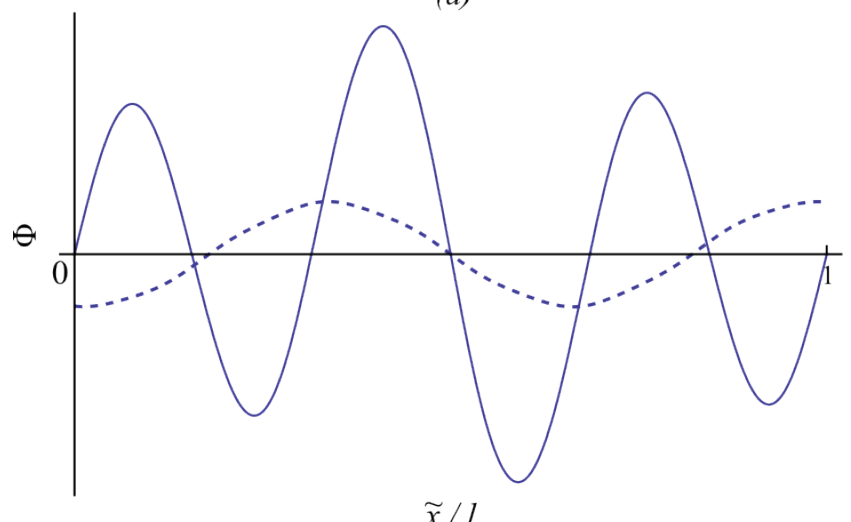

(c)

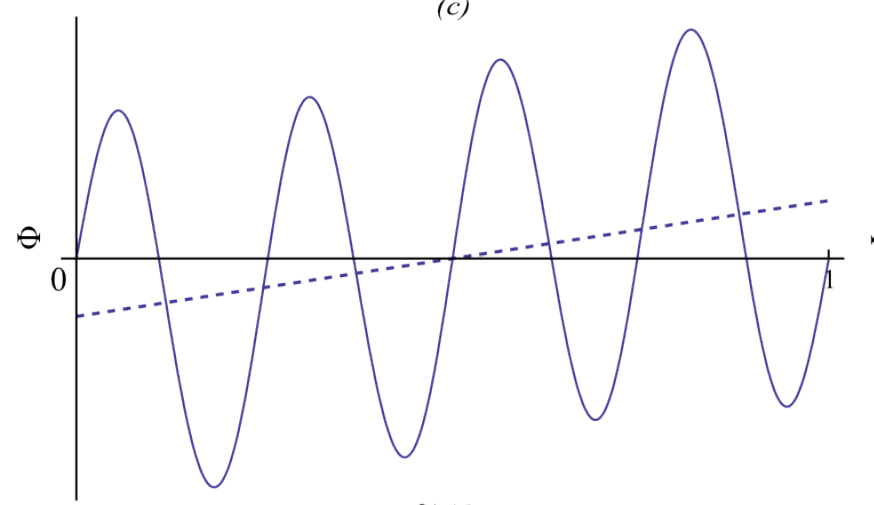

$\tilde{x} / l$

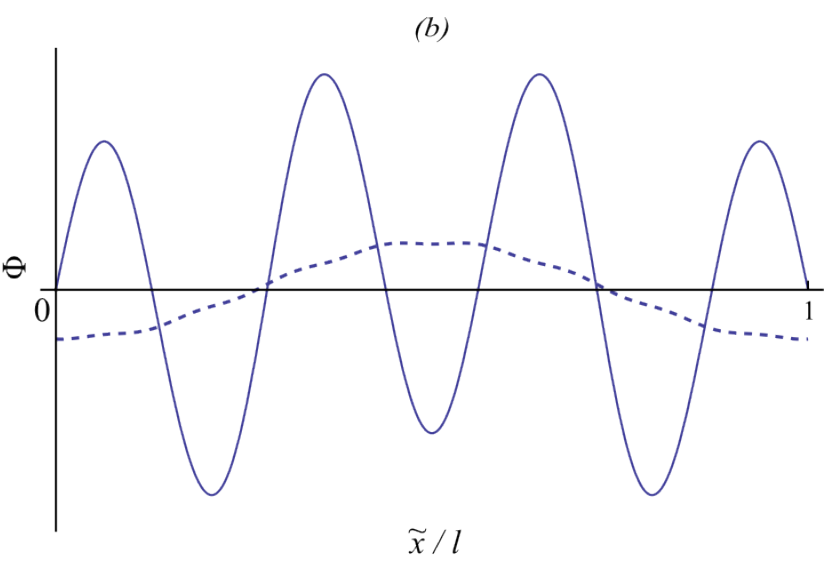

(d)

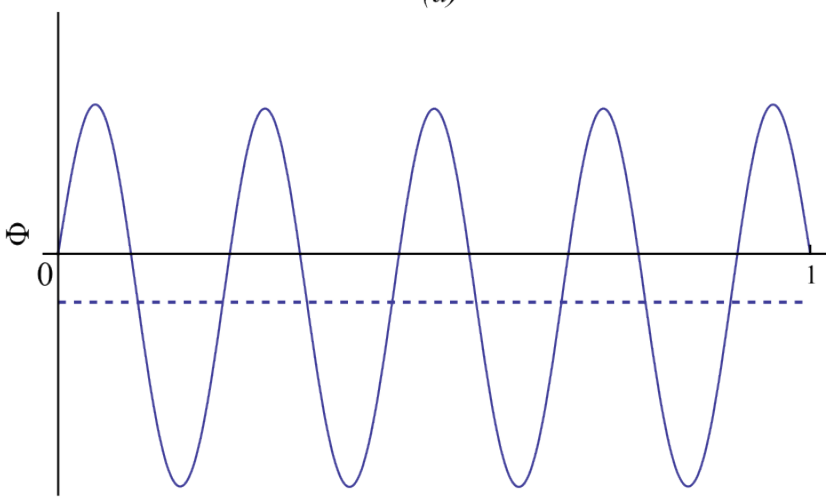

$\tilde{x} / l$

(e)

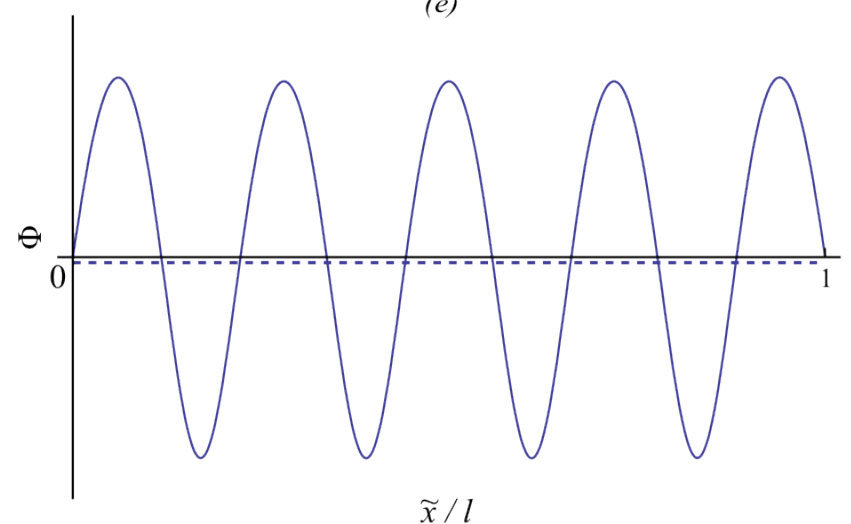

$\tilde{x} / l$

Figure 6. The sixth, seventh, eighth, and ninth eigenmodes of the non-uniform beam for parameters (27) and (a)-(d) $\chi_{A}=0.5, \chi_{I}=0$; (e) $\chi_{A}=0, \chi_{I}=0.5$. Dashed line represents the long-wave component of the eigenmodes.

\section{Validation by numerical experiments}

A series of numerical experiments was conducted to validate the analytical results obtained in Sections 4 and 5. The values of $\delta$ providing nonzero solutions of (8) for given boundary conditions (9) were determined using Wolfram Mathematica 7 (NDSolve). Consequently, the eigenfrequencies and eigenmodes of the considered non-uniform beam were obtained numerically, for comparison with the determined analytical solution (23), (25).

Figure 7 shows the relative deviation between analytical and numerical values of the eigenfrequencies for parameters (26) and various modulation amplitudes $\chi_{A}, \chi_{I}$. As appears there 
is good agreement between analytical and numerical results. The same agreement is present also for parameters (27) considered in Section 5.2. Thus, the effects of cross-section modulation on the beam eigenfrequencies, revealed in Section 5, are validated.

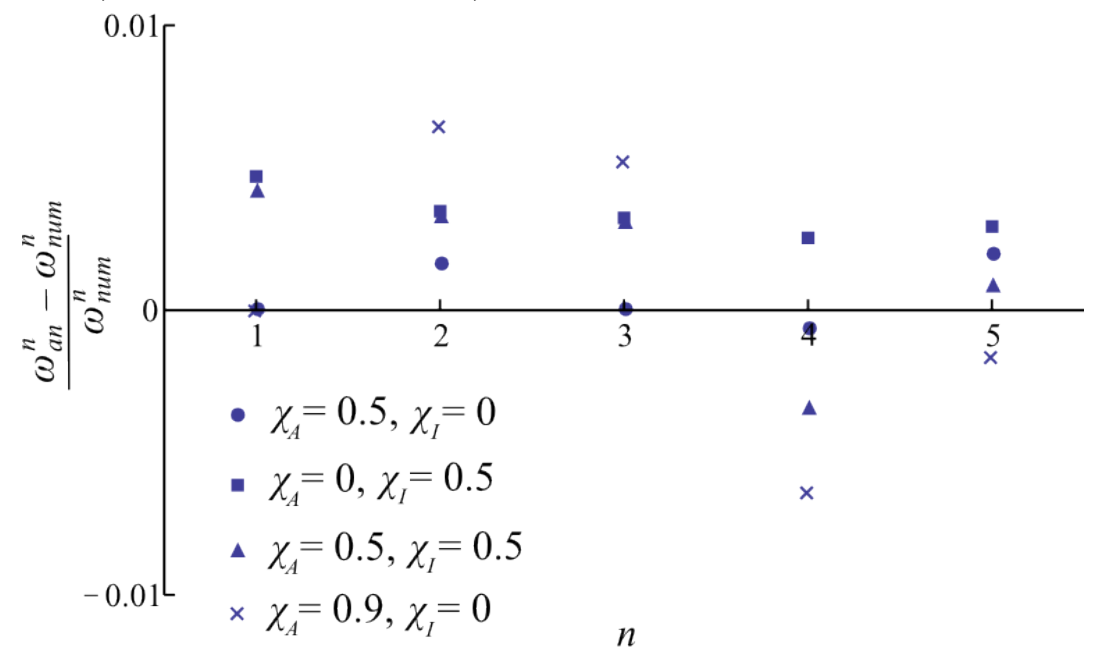

Figure 7. Relative deviation between analytical $\omega_{a n}^{n}$ and numerical $\omega_{n u m}^{n}$ eigenfrequencies for parameters (26).

The revealed effects of modulation on eigenmodes of the beam were also validated by the numerical experiments. As an illustration, the eigenmodes are presented in Figure 8 for parameters (26) and various modulation amplitudes $\chi_{A}, \chi_{I}$; here solid lines represent numerical and dashed lines analytical results; as appears they are in good agreement. Thus in particular the emergence of a long-wave component in eigenmodes having a period close to the modulation period is confirmed. 
(a)

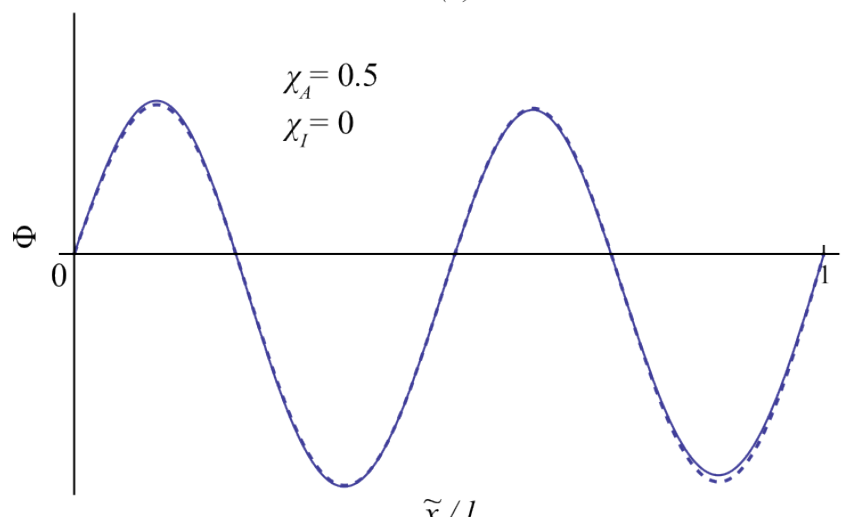

(c)

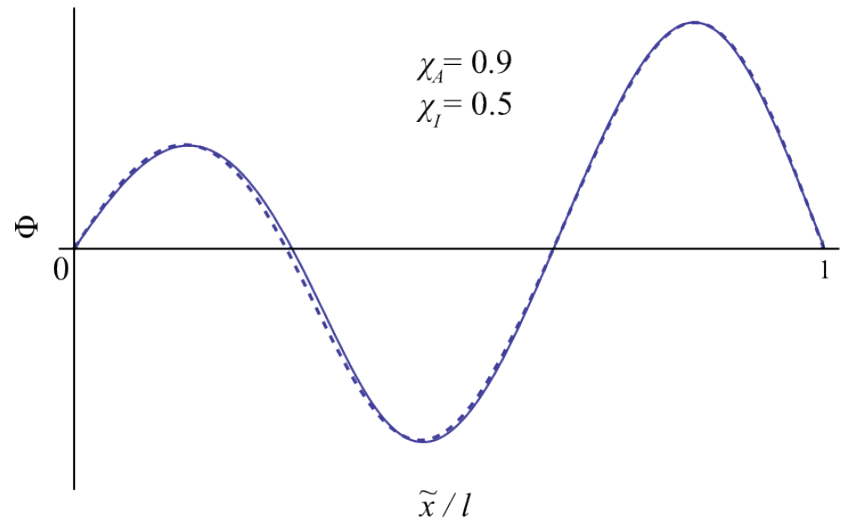

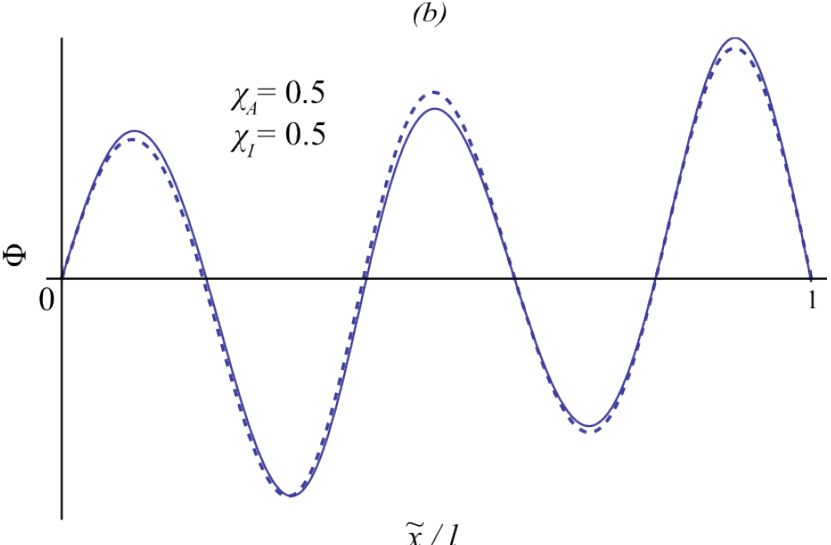

(d)

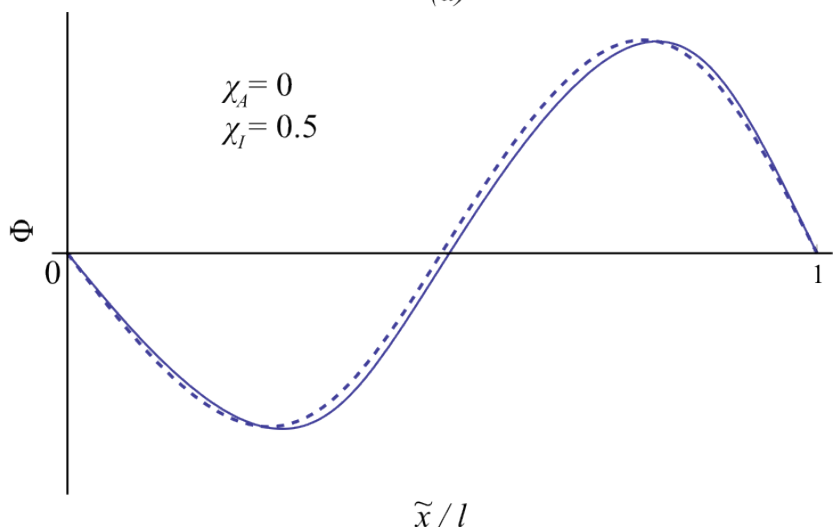

Figure 8. Eigenmodes of the non-uniform beam for parameters (26) and various modulation amplitudes $\chi_{A}, \chi_{I}$; solid lines represent numerical and dashed lines analytical results.

\section{Conclusions}

The paper addresses the prediction of eigenfrequencies and eigenmodes of a beam with periodically continuously varying cross-section. The effects of modulation on beam eigenproperties are studied analytically. Special attention is given to "mid-frequency" eigenmodes, having a spatial period of the same order as the period of modulation, which cannot be captured by conventional analytical methods, e.g. [15, 19].

A dispersion relation of the considered non-uniform periodic structure is obtained, and values of the modulation amplitudes at which frequency bandgaps arise are determined. It was found that pure modulation of the beam mass per unit length leads to the emergence of two distinct bandgaps in the considered frequency range, while pure modulation of the beam stiffness or modulations with equal amplitudes lead to a single bandgap. It was observed that eigenfrequencies of the beam can lie within the bandgaps, and that such eigenfrequencies can be considerably affected by modulation. In particular, for non-zero modulation of the beam mass per unit length, the eigenfrequency corresponding to an eigenmode with a period equal to the modulation period is always within the bandgap, and is increased considerably by modulation.

It was found that there is an abrupt shift in the effect of modulation on the eigenfrequencies corresponding to eigenmodes having a period of the same order as the period of modulation: comparatively low eigenfrequencies are changed in one way, and higher eigenfrequencies in 
another. It was shown also that the effects of modulations of the beam mass per unit length and of the beam stiffness are opposite. Thus, if both types of modulations are imposed, the eigenfrequencies which correspond to eigenmodes having a period of the same order as the period of modulation can be either reduced or increased. The effects revealed clearly indicate the capability of cross-section modulation to change the spacing between two consecutive eigenfrequencies, especially the one for which the detected abrupt shift is present. A relation between the index of this spacing and the period of modulation is determined. It is noted, however, that if modulations of the beam mass per unit length and of the beam stiffness have equal amplitudes, then no abrupt shift in the effect of modulation is noticed.

Another effect, noted apparently for the first time, is the presence of a long-wave component in eigenmodes having a period close to the period of modulation; for an eigenmode having a period equal to the modulation period this component is almost constant. An explanation of this effect is given, showing its relation to the dispersion relation. This effect seems to be rather important, since it illustrates the capacity of non-uniform structures to sustain long-wave oscillations at comparatively high frequencies. The analytical results are validated by a series of numerical experiments, in all cases showing good agreement.

\section{Acknowledgments}

The work is carried out with financial support from the Danish Council for Independent Research and FP7 Marie Curie Actions - COFUND: DFF - 1337-00026.

\section{References}

[1] Kirchhoff, G., 1879. Über die transversalschwingungen eines stabes von veränderlichem quershnitt. Berl Monatsberichte, 10, pp. 815-828.

[2] Ward, P.F., 1913.Transverse vibration of bars of varying cross section. Philos Mag, 25, pp. 85106.

[3] Nicholson, J.W., 1917. The lateral vibrations of bars of variable section. Proc $R$ Soc, 93, pp. 506-519.

[4] Lenci, S., Clementi, F., Mazzilli, C.E.N., 2013. Simple formulas for the natural frequencies of non-uniform cables and beams. Int J Mech Sci, 77, pp. 155-163.

[5] Conway, H.D., Dubil, J.F., 1965. Vibration frequencies of truncated cones and wedge beams. ASME J Appl Mech, 31, pp. 329-331.

[6] Mabie, H., Rogers, C., 1972. Transverse vibrations of double-tapered cantilever beams. J Acoust Soc Am, 51, pp. 1771-1774.

[7] Williams, F.W., Banerjee, J.R., 1985. Flexural vibration of axially loaded beams with linear or parabolic taper. J Sound Vib, 99, pp. 121-138.

[8] Shahba, A., Rajasekaran, S., 2012. Free vibration and stability of tapered Euler-Bernoulli beams made of axially functionally graded materials. Appl Math Model, 36, pp. 3094-111.

[9] Goel, R.P., 1976. Transverse vibrations of tapered beam. J Sound Vib, 47, pp. 1-7.

[10] Abrate, S., 1995. Vibration of non-uniform rods and beams. J Sound Vib, 185, pp. 703-716.

[11] De Rosa, M.A., Auciello, N.M., 1996. Free vibrations of tapered beams with flexible ends. Comput Struct, 60, pp. 197-202.

[12] Auciello, N.M., Nolè, G., 1998. Vibrations of a cantilever tapered beam with varying section properties and carrying a mass at the free end. J Sound Vib, 214, pp. 105-119. 
[13] Grossi, R.O., Bhat, R.B., 1991. A note on vibrating tapered beams. J Sound Vib, 147, pp. 174178.

[14] Tarnopolskaya, T., de Hoog, F., Fletcher, N.H., Thwaites, S., 1996. Asymptotic analysis of the free in-plane vibrations of beams with arbitrarily varying curvature and cross-section. $J$ Sound Vib, 196, pp. 659-680.

[15] Pierce, A.D., 1970. Physical interpretation of the WKB or Eikonal approximation for waves and vibrations in inhomogeneous beams and plates. $J$ Acoust Soc Am, 48, pp. 275-284.

[16] Firouz-Abadi, R.D., Haddadpour, H., Novinzadeh, A.B., 2007. An asymptotic solution to transverse free vibrations of variable-section beams. J Sound Vib, 304, pp. 530-540.

[17] Shu, Qi.G., Shao, Pu.Y., 2011. Free longitudinal vibrations of non-uniform rods. Sci China Tech Sci, 10, pp. 2735-2745.

[18] Nielsen, R., Sorokin, S., 2014. The WKB approximation for analysis of wave propagation in curved rods of slowly varying diameter. Proc R Soc A, 470: 20130718.

[19] Bakhvalov, N.S., Panasenko, G.P., 1989. Homogenization: Averaging Processes in Periodic Media, Kluwer.

[20] Chapman, C.J., Sorokin, S.V., 2010. The finite-product method in the theory of waves and stability. Proc $R$ Soc A, 466, pp. 471-491.

[21] Olhoff, N., Niu, B., Cheng, G., 2012. Optimum design of band-gap beam structures. Int J Solid Struct, 49, pp. 3158-3169.

[22] Brillouin, L., 1953. Wave Propagation in Periodic Structures second edition, Dover Publications.

[23] Sorokin, V.S., Thomsen, J.J., 2015. Vibration suppression for strings with distributed loading using spatial cross-section modulation. J Sound Vib, 335, pp. 66-77.

[24] Blekhman, I.I., 2000. Vibrational Mechanics, World Scientific.

[25] Blekhman, I.I., 2004. Selected Topics in Vibrational Mechanics, World Scientific.

[26] Bolotin, V.V, 1964. The Dynamic Stability of Elastic Systems, Holden-Day.

[27] Bogoliubov, N.N., Mitropolskii, Ju.A., 1961. Asymptotic Methods in the Theory of Non-linear Oscillations, Gordon and Breach.

[28] Sanders, J.A., Verhulst, F., 1985. Averaging Methods in Nonlinear Dynamical Systems, Springer-Verlag.

[29] Nayfeh, A.H., Mook, D.T., 1979. Nonlinear Oscillations, Wiley-Interscience.

[30] Yakubovich, V.A., Starzhinskii, V.M., 1975. Linear Differential Equations with Periodic Coefficients, John Wiley \& Sons.

[31] Mead, D.J., 1970. Free wave propagation in periodically supported, infinite beams. J Sound Vib, 11, pp. 181-197.

[32] Sorokin, V.S., 2014. On the unlimited gain of a nonlinear parametric amplifier. Mech. Re. Commun., 62, pp. 111-116.

[33] Sorokin, V.S., 2013. A new approach to the analysis of oscillations of one-dimensional spatially periodic structures. J Sound Vib, 332, pp. 3552-3563. 